\title{
Bio-enhanced Curcumin Extract Formulation
}

\author{
National Cancer Institute
}

\section{Source}

National Cancer Institute. Bio-enhanced Curcumin Extract Formulation. NCI Thesaurus. Code C161600.

\begin{abstract}
A bio-enhanced extract-based preparation containing curcumin, a poorly water-soluble phytopolylphenol pigment isolated from the plant Curcuma longa, commonly known as turmeric, with a variety of pharmacologic properties, including antineoplastic, chemopreventive, antioxidant, anti-angiogenic, neuroprotective and anti-inflammatory activities. Upon oral administration, curcumin blocks the formation of reactive-oxygen species, neutralizes free radicals, and prevents oxidative stress and DNA damage. Curcumin also possesses anti-inflammatory properties as a result of inhibition of cyclooxyg enases (COX) and other enzymes involved in inflammation. It disrupts various cell signal transduction pathways involved in carcinogenesis and prevents the expression of many transcription factors. In addition, curcumin modulates phase II enzymes. Altogether, this prevents and/or inhibits tumor cell formation and proliferation. Compared to the very poorly absorbed curcumin alone, this extract formulation, by micronizing curcumin and adding turmeric essential oils, has a much greater bioavailability, an improved pharmacokinetic profile and increased efficacy.
\end{abstract}

